\title{
Updating and downdating of orthonormal polynomial vectors and some applications
}

\author{
Marc Van Barel and Adhemar Bultheel
}

\begin{abstract}
In this paper we develop procedures to update and downdate the recurrence information for sequences of polynomial vectors orthonormal with respect to a discrete inner product. The usefulness of these algorithms is shown by using them for data fitting, for solving a system of polynomial equations and for solving a Bezout equation.
\end{abstract}

\section{Introduction}

Given the complex points $z_{i} \in \mathbb{C}$ and weights $w_{i}>0$ for $i=1, \ldots, m$ consider the inner product $\langle f, g\rangle=\sum_{i=1}^{m} \overline{f\left(z_{i}\right)} g\left(z_{i}\right) w_{i}$. For polynomial least squares problems with respect to this inner product, Elhay, Golub and Kautsky [9] describe several updating and downdating procedures for orthogonal polynomials (together with the coefficients for the least squares fit) when the points $z_{i}$ are on the real line. One of the procedures uses orthogonal similarity transformations where the transformation is achieved by rotations instead of elementary Householder matrices. This idea can be traced back to Rutishauser [16] and can be found more recently in Gragg and Harrod [11] and Boley and Golub [6]. For the block case, we refer to Ammar and Gragg [2].

When the $z_{i}$ are not on the real line but on the unit circle, Ammar, Gragg and Reichel [4] construct a downdating procedure using the Schur parametrization of the corresponding unitary Hessenberg matrix. This is a continuation of the ideas developed by Ammar and He [1] and Ammar, Gragg and Reichel [3].

For linearized discrete rational least squares approximation, we have to generalize the inner product to polynomial vectors as in (1) below. In [21], we developed an updating procedure to compute a sequence of orthonormal polynomial vectors with respect to that inner product where the points $z_{i}$ could lie anywhere in the $41 \mathrm{~A} 20$.

1991 Mathematics Subject Classification. Primary 42C05; Secondary 30E10, 65D10, 41A28,

Key words and phrases. orthogonal systems, discrete least squares approximation, updating, downdating.

This work is partially supported by the Fund for Scientific Research (FWO), project "Orthogonal systems and their applications", grant \#G.0278.97 and the Belgian Programme on Interuniversity Poles of Attraction, initiated by the Belgian State, Prime Minister's Office for Science, Technology and Culture. The scientific responsibility rests with the authors. 
complex plane. When they are on the real line or on the unit circle, the amount of computational work decreases by an order of magnitude. This generalizes the methods based on unitary similarity transformations mentioned above. We also gave a least squares approximation interpretation of these orthonormal polynomial vectors.

In this paper, we construct the corresponding downdating procedures for orthonormal polynomial vectors.

To illustrate the usefulness of the orthonormal polynomial vectors, we use them to solve a data fitting problem, to solve a system of 2 polynomial equations in 2 variables and to solve a Bezout equation.

\section{Sequence of orthonormal polynomial vectors}

We consider the following inner product.

Definition 2.1 (inner product, norm). Given the points $z_{i} \in \mathbb{C}$, and the weight vectors $F_{i} \in \mathbb{C}^{1 \times n}, i=1,2, \ldots, m$, we consider a subspace $\mathcal{P} \subset \mathbb{C}[z]^{n \times 1}$ of polynomial vectors such that the following bilinear form defines a discrete inner product $\langle P, Q\rangle$ for two polynomial vectors $P, Q \in \mathcal{P} \subset \mathbb{C}[z]^{n \times 1}$ :

$$
\langle P, Q\rangle:=\sum_{i=1}^{m} P^{H}\left(z_{i}\right) F_{i}^{H} F_{i} Q\left(z_{i}\right) .
$$

The norm $\|P\|$ of a polynomial vector $P \in \mathcal{P} \subset \mathbb{C}[z]^{n \times 1}$ is defined as:

$$
\|P\|:=\sqrt{\langle P, P\rangle} \text {. }
$$

For this to be an inner product in $\mathcal{P}$, it is necessary and sufficient that $\mathcal{P}$ is a subspace of polynomial vectors such that there is no nonzero polynomial vector $P \in \mathcal{P}$ with $\langle P, P\rangle=0$ or equivalently with $F_{i} P\left(z_{i}\right)=0, i=1,2, \ldots, m$. We will call this the regular case.

In [21], we developed an updating procedure to compute a sequence of orthonormal polynomial vectors with respect to the inner product (1).

The initial scheme

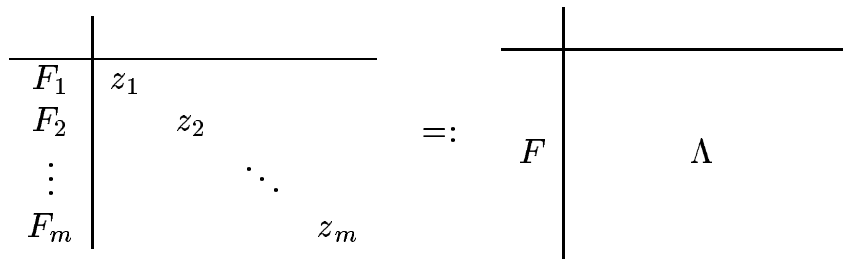

is transformed, using unitary similarity transformations on $\Lambda$, into

$$
\left[Q^{H} F \mid Q^{H} \Lambda Q\right]=Q^{H}[F \mid \Lambda]\left[\begin{array}{ll}
I_{n} & \\
& Q
\end{array}\right]
$$

( $Q$ unitary) such that in the simplest case $[E \mid G]:=\left[Q^{H} F \mid Q^{H} \Lambda Q\right]$ is upper trapezoidal, i.e., has zeros below the main diagonal positions $(i, i)$. In general, however, this is only true up to a column permutation. This means that there exists a permutation $\Pi=\left(\pi_{1}, \pi_{2}, \ldots, \pi_{m}\right)$ of the numbers $(1,2, \ldots, m)$ such that the matrix $[E \mid G]$ has zeros below the pivot positions $\left(i, \pi_{i}\right), i=1,2, \ldots, m$. The pivot indices $\pi_{i}$ will determine the degree structure of the associated sequence of orthonormal polynomial vectors as indicated in Section 6 . The unitary transformation 
matrix $Q$ is built up recursively. In step $i(1 \leq i \leq m)$, the set $\left\{\pi_{j} \mid j=1,2, \ldots, i\right\}$, satisfies

$$
\left\{\pi_{j} \mid j=1,2, \ldots, i\right\}=\{1,2, \ldots, k\} \cup\{n+1, n+2, \ldots, n+l\}
$$

with $k \leq n$ and $k+l=i$. Hence, if $i<m$ and $k<n, \pi_{i+1}$ has one of the two values: $k+1$ or $n+l+1$. Without loss of generality, we can always take the first pivot index $\pi_{1}=1$. Hence, if $\pi_{i}>n$, then $\pi_{i}<i+n$, i.e. the pivot position $\left(i, \pi_{i}\right)$ lies below the main diagonal of $G$.

The following algorithm performs the similarity transformation:

Algorithm 2.2. Transformation of the initial data matrix $D:=[F \mid \Lambda]$ into a matrix $[E \mid G]:=\left[Q^{H} F \mid Q^{H} \Lambda Q\right]$ having zeros below the pivot elements

$$
\begin{aligned}
& \text { for } i:=1 \text { to } m \text { do } \\
& \text { for } j:=1 \text { to } i-1 \text { do } \\
& * \text { make element } d_{i, \pi_{j}} \text { zero } \\
& \text { by using a Givens rotation (or reflection) } J^{H} \\
& \text { with the pivot element }\left(j, \pi_{j}\right) \text { : } \\
& \quad D \leftarrow J^{H} D \\
& \quad * \quad D \leftarrow D\left[\begin{array}{ll}
I_{n} & \\
& J
\end{array}\right] \text { (similarity transformation) }
\end{aligned}
$$

Execution of this algorithm requires $O\left(m^{3}\right)$ FLOPS. In Section 5, we show that this amount can be reduced by an order of magnitude when the points $z_{i}$ are all on the real line or all on the unit circle.

If we denote by $P_{\pi}$ the $(m+n) \times m$ matrix selecting the columns $\pi_{1}, \pi_{2}, \ldots, \pi_{m}$ in that order, we know that $[E \mid G] P_{\pi}$ is an $m \times m$ upper triangular matrix. Hence, the equality

$$
[F \mid \Lambda Q] P_{\pi}=Q[E \mid G] P_{\pi}
$$

is a $Q R$ factorization and, hence, gives us a forward recurrence relation for the columns of $Q$.

We construct the recurrence relation for the orthonormal polynomial vectors such that the orthonormality of these vectors is equivalent to $Q$ being unitary. Therefore, we compute the polynomial vectors $\phi_{k}(z) \in \mathbb{C}[z]^{n \times 1}, k=0,1, \ldots, m-1$, by a forward recurrence relation from the equality

$$
\left[I_{n} \mid z\left[\phi_{0}(z), \phi_{1}(z), \ldots \phi_{m-1}(z)\right]\right] P_{\pi}=\left[\phi_{0}(z), \phi_{1}(z), \ldots \phi_{m-1}(z)\right][E \mid G] P_{\pi} .
$$

Filling in a value for $z$, we can use (4) to compute the value of the orthonormal polynomial vectors. From (3) and (4), it follows that

$$
Q_{k, i}=F_{i} \phi_{k}\left(z_{i}\right)
$$

and because $Q^{H} Q=I_{m}$ we have that the $\phi_{k}(z)$ are orthonormal w.r.t. the inner product (1).

Because $[E \mid G]$ contains all the information for the computation of the orthonormal polynomial vectors $\phi_{k}(z)$, we will call this the recurrence data matrix. 


\section{Downdating}

Downdating transforms the recurrence data matrix $[E \mid G]$ by a similarity transformation on $G$ into

$$
Q[E \mid G]\left[\begin{array}{ll}
I_{n} & 0 \\
0 & Q^{H}
\end{array}\right]=:\left[\begin{array}{l|ll}
\underline{E} & \underline{G} & 0 \\
F_{i} & \frac{z_{i}}{0}
\end{array}\right]
$$

where $z_{i}$ is one of the eigenvalues of $G$ and $F_{i}$ is the corresponding weight vector. The smaller recurrence data matrix $[\underline{E} \mid \underline{G}]$ still has zero elements below the pivot elements.

From (5), it follows that the last column $p$ of $Q^{H}$ is a right and $p^{H}$ a left eigenvector of $G$ corresponding to the eigenvalue $z_{i}$ and such that $p^{H} E=F_{i}$. Because $[E \mid G] P_{\pi}$ is upper triangular, it is easy to find $p$ by forward substitution from

$$
p^{H}\left[E \mid G-z_{i} I_{m}\right] P_{\pi}=\left[F_{i} \mid 0^{H}\right] P_{\pi} .
$$

Hence, if we take $Q$ such that $Q p=e_{m},(5)$ is satisfied but $[\underline{E} \mid \underline{G}]$ not necessarily has the correct zero pattern. To ensure that the correct zero pattern is maintained, we build up $Q$ as a product of Givens rotations starting with $G_{m-1}$

$$
Q=G_{1} G_{2} \cdots G_{m-2} G_{m-1}
$$

where $G_{j}$ is the Givens rotation making the element $j$ of the vector $p$ zero based on the elements $j$ and $m$ of $p$. This leads to the following downdating algorithm.

Algorithm 3.1. downdate $\left(m, F_{i}, z_{i}\right)$

Compute $p$ using forward substitution from $p^{H}\left[E \mid G-z_{i} I_{m}\right] P_{\pi}=\left[F_{i} \mid 0^{H}\right] P_{\pi}$. for $j:=m-1$ downto 1 do

* $G_{j}$ is the Givens rotation such that the element $j$ of $p$ is eliminated based on the elements $m$ and $j$ of $p$.

* $p \leftarrow G_{j} p$

$*[E \mid G] \leftarrow G_{j}[E \mid G]\left[\begin{array}{ll}I_{n} & 0 \\ 0 & G_{j}^{H}\end{array}\right]$

Note that after each $j$-step, we have

$$
\left(G-z_{i} I_{m}\right) p=0 .
$$

From the $k$-th row of this equality, it follows that if $\pi_{k}=n+j$ with $k<m, G_{j}^{H}$ is also the Givens rotation making the element $(k, m)$ of $G$ zero based on the two elements $(k, m)$ and $\left(k, \pi_{k}-n\right)$ of $G$. This leads to another possibility to determine the Givens rotation $G_{j}$ for $j$ small enough such that $\pi_{k}=n+j$ with $k<m$. To refer to this variant, we will use the notation variantdowndate.

Algorithm 3.2. variantdowndate $\left(m, F_{i}, z_{i}\right)$

Compute $p$ using forward substitution from $p^{H}\left[E \mid G-z_{i} I_{m}\right] P_{\pi}=\left[F_{i} \mid 0^{H}\right] P_{\pi}$.

for $j:=m-1$ downto 1 do

* if $\pi_{k}=n+j$ and $k<m$ then

$G_{j}$ is the Givens rotation such that the element $(k, m)$ of $G$ is eliminated based on the elements $(k, m)$ and $\left(k, \pi_{k}-n\right)$ of $G$.

else

$G_{j}$ is the Givens rotation such that the element $j$ of $p$ is eliminated

based on the elements $m$ and $j$ of $p$.

$* p \leftarrow G_{j} p$ 
$*[E \mid G] \leftarrow G_{j}[E \mid G]\left[\begin{array}{ll}I_{n} & 0 \\ 0 & G_{j}^{H}\end{array}\right]$

Another way to write this down is the following. One starts from the recurrence data matrix $[E \mid G]$ and transforms this matrix into the following form using a similarity transformation on $G$

$$
Q[E \mid G]\left[\begin{array}{ll}
I_{n} & 0 \\
0 & Q^{H}
\end{array}\right]=:\left[\begin{array}{l|ll}
F_{i} & z_{i} & 0 \\
\underline{E} & 0 & \underline{G}
\end{array}\right]
$$

where $[\underline{E} \mid \underline{G}]$ has the desired zero pattern, i.e. zeros under the pivot elements. In this case $p$ is the first column of $Q^{H}$. The downdating procedure goes as follows:

Algorithm 3.3. anotherdowndate $\left(m, F_{i}, z_{i}\right)$

Compute $p$ using forward substitution from $p^{H}\left[E \mid G-z_{i} I_{m}\right] P_{\pi}=\left[F_{i} \mid 0^{H}\right] P_{\pi}$. for $j:=m$ downto 2 do

* $G_{j}$ is the Givens rotation such that the element $j$ of $p$ is made equal to zero based on the elements $j-1$ and $j$ of $p$.

* $p \leftarrow G_{j} p$

$*[E \mid G] \leftarrow G_{j}[E \mid G]\left[\begin{array}{ll}I_{n} & 0 \\ 0 & G_{j}^{H}\end{array}\right]$

Also here, after each $j$-step, we have

$$
\left(G-z_{i} I_{m}\right) p=0 .
$$

From this equality, it follows that $[\underline{E} \mid \underline{G}]$ has the desired zero pattern. We prove this by induction on $j$ from Algorithm 3.3. Suppose that after the transformation with $G_{j+1}$ the zero pattern of $[E \mid G]$ is the following for $i=1,2, \ldots, m-1$ :

- if $i<j$, there are zeros below position $\left(i, \pi_{i}\right)$;

- if $i \geq j$,

- if $\pi_{i}<j+n$, there are zeros below position $\left(i+1, \pi_{i}\right)$;

- if $\pi_{i} \geq j+n$, there are zeros below $\left(i+1, \pi_{i}+1\right)$;

- there are zeros below $(k+1, j)$ with $\pi_{k}=j-1+n$.

Note that this is the zero structure at the start of the algorithm with $j=m$. Applying the similarity transformation based on $G_{j}$, we get the zero pattern as indicated above with $j-1$ substituted for $j$ except for column $j-1$ which has zeros below $(k+1, j-1)$ with $\pi_{k}=j-1+n$. However, with $l$ defined as $\pi_{l}=j-2+n$, we know from rows $l+2, l+3, \ldots, k+1$ from equality (8) together with the fact that $p_{j}, p_{j+1}, \ldots, p_{m}$ is zero, that the elements $(i, j-1), i=l+2, l+3, \ldots, k+1$ are zero. Hence, there are also zeros below $(k+1, j-1)$ with $k$ defined by $\pi_{k}=j-2+n$. Note that the Givens rotation $G_{j}^{H}$ can also be defined by making element $(k+1, j-1)$ zero based on the two elements $(k+1, j-1)$ and $(k+1, j)$. For $j=2$, we get the desired zero pattern for $[\underline{E} \mid \underline{G}]$.

Note that execution of Algorithm 3.1, 3.2 or 3.3 requires $O\left(m^{2}\right)$ FLOPS.

\section{Downdating when the recurrence data matrix is only partially known}

In several applications, we do not need the complete recurrence data matrix but only the part determining the first $l$ orthonormal polynomial vectors $\phi_{k}(z)$. Then, we do not update or donwdate the complete recurrence data matrix $[E \mid G]$ 
but only the principal $l \times(n+l)$ part $[\underline{E} \mid \underline{G}]$. This part contains enough recurrence information to compute the first $l$ orthonormal polynomial vectors $\phi_{k}(z)$, $l=0,1, \ldots, l-1$.

If we knew the complete recurrence data matrix $[E \mid G]$, then after downdating using Algorithm 3.1 or 3.2 based on the elements $m-1, m-2, \ldots, l+1$ of the original eigenvector $p$, we would have the following equality

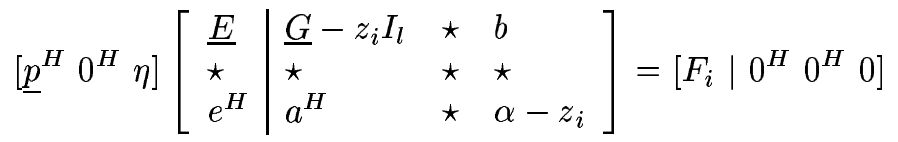

where the partitioning is $l, m-l-1,1$. The entries indicated by $\star$ will not influence the other entries in the sequel of the downdating procedure. The matrix $[\underline{E} \mid \underline{G}]$ is the original principal part of the full recurrence data matrix. Hence, instead of doing the first part of the downdating procedure, we can compute the entries $e^{H}, a^{H}, \alpha, b, \underline{p}, \eta$ such that $\left[\underline{p}^{H} \eta\right]$ with norm one is a left and the complex conjugate transpose is a right eigenvector of the matrix

$$
\left[\begin{array}{ll}
\frac{G}{a^{H}} & b
\end{array}\right]
$$

for the eigenvalue $z_{i}$ satisfying

$$
\left[\underline{p}^{H} \eta\right]\left[\frac{\underline{E}}{e^{H}}\right]=F_{i} .
$$

The first part $p$ of the eigenvector can be computed by forward substitution from the set of linear equations

$$
\underline{p}^{H}\left[\underline{E} \mid \underline{G}-z_{i} I_{l}\right] P_{\pi}=\left[F_{i} \mid 0^{H}\right] P_{\pi} .
$$

We compute $\eta$ such that the norm of the eigenvector is one. Hence, $\eta$ is nothing but the norm of the vector consisting of the last $m-l$ elements of the original eigenvector $p$ (up to the sign). Once we have $\underline{p}$ and $\eta$, we can compute $a$ and $e$ from

$$
\eta\left[\begin{array}{ll}
e^{H} & a^{H}
\end{array}\right]=-\underline{p}^{H}\left[\begin{array}{ll}
\underline{E} & \underline{G}-z_{i} I_{l}
\end{array}\right]+\left[\begin{array}{ll}
F_{i} & 0
\end{array}\right] .
$$

Finally, $b$ and $\alpha$ can be computed from

$$
\left[\begin{array}{l}
b \\
\alpha-z_{i}
\end{array}\right] \eta=-\left[\frac{G}{a^{H}}-z_{i} I_{l}\right] \underline{p} .
$$

The last part of the downdating procedure on the full original recurrence data matrix is equivalent to applying the downdating procedure to the matrix

$$
\left[\begin{array}{l|ll}
\underline{E} & \frac{G}{e^{H}} & b \\
a^{H} & \alpha
\end{array}\right]
$$

based on the eigenvector $\left[\underline{p}^{H} \eta\right]$. Note that the recurrence data matrix (9) has the desired zero pattern.

\section{Special cases}

In this section, we indicate how the amount of computational work can be reduced by an order of magnitude when all points $z_{i}$ are on the real line or all on the unit circle. 
5.1. All points $z_{i}$ are real. If $z_{i} \in \mathbb{R}, i=1,2, \ldots, m$, then $G:=Q^{H} \Lambda Q$ is Hermitian because

$$
G^{H}=\left(Q^{H} \Lambda Q\right)^{H}=Q^{H} \Lambda Q=G .
$$

Because $g_{k, j}=0, j<\pi_{k}$, also $g_{j, k}=0, j<\pi_{k}$. Hence, the nonzero elements of $G$ are located around the main diagonal and this zero structure can be exploited to reduce the complexity $O\left(\mathrm{~m}^{3}\right)$ FLOPS of Algorithm 3.1 downdate, 3.2 variantdowndate (and 3.3 anotherdowndate to $O\left(\mathrm{~m}^{2}\right)$ FLOPS. This is done in a similar way as explained in [21] for the updating procedure. We denote these more efficient algorithms by downdatereal and variantdowndatereal, repsectively.

5.2. All points $z_{i}$ are on the unit circle. If $\left|z_{i}\right|=1, i=1,2, \ldots, m$, then $G:=Q^{H} \Lambda Q$ is a unitary block Hessenberg matrix. This does not lead to additional zeros like in the real case but the updating and downdating algorithms can be rewritten using a decomposition of the matrix $G$.

Theorem 5.1 (generalized block Schur parameter decomposition [21]). The unitary block Hessenberg matrix $G:=Q^{H} \Lambda Q$ can be decomposed as

$$
G=G_{1} G_{2} G_{3} \ldots G_{m-\tau_{m}}
$$

with $G_{i}$ having the form

$$
G_{i}:=\left[\begin{array}{lll}
I_{k-1} & & \\
& G_{i}^{\prime} & \\
& & I_{m-k-1-\lambda_{i}}
\end{array}\right]
$$

with $\tau_{k}:=k+n-\pi_{k}=\#\left\{\pi_{j} \mid 1 \leq \pi_{j} \leq n, j<k\right\}$ and $G_{i}^{\prime}$ a unitary $\left(\lambda_{i} \times \lambda_{i}\right)$-matrix (block Schur parameters) where $\lambda_{i}:=\tau_{k}+1$ with $k$ such that $\pi_{k}=i+n$.

Instead of using the unitary block Hessenberg matrix $G$ in the updating and downdating procedures, we transform the blocks $G_{i}^{\prime}$ of the block Schur parametrization of $G$. This reduces the order of computations by a factor $m$. For further details we refer to $[\mathbf{2 1}]$.

\section{Least squares approximation}

Suppose we want to approximate a set of data points $\left(z_{i}, f_{i}, e_{i}\right) \in \mathbb{C}^{3}, i=$ $1,2, \ldots, m$, by a rational function $n(z) / d(z)$ of a given degree structure in the sense that we want to minimize the sum of squares $\tilde{S}$ with

$$
\tilde{S}:=\sum_{i=1}^{m}\left|f_{i} / e_{i}-n\left(z_{i}\right) / d\left(z_{i}\right)\right|^{2} .
$$

This is a highly nonlinear problem which requires an iterative solver. See Wittmeyer [23], Barrodale and Mason [5], or Spellucci [18]. Moreover, it is not guaranteed that a unique (global) minimizer exists [18]. Consider a simple example where the approximant is of the form $n_{0} /\left(1+d_{1} z\right)$ which has to fit the data $\left(z_{i}, f\left(z_{i}\right)\right)$ given by

- $(-1,-1),(1,1)$, then there is no local minimizer;

- $(1,1),(2,5 / 12),(4,5 / 12)$, then there are two local minima of which one is global. 
Other situations may occur. However, in the case of real $z_{i}$ and if the data are close to the exact data of a model that is of the correct degree structure and if it has no poles in the interval where the $z_{i}$ are chosen, then Pomentale [13] proved that an optimal solution exists.

A good starting value for the iteration can often be obtained by solving the linearized problem, where we minimize $S$ with

$$
S:=\sum_{i=1}^{m}\left|f_{i} d\left(z_{i}\right)-e_{i} n\left(z_{i}\right)\right|^{2} .
$$

The latter problem is linear and much easier to solve. Note that (10) and (11) are similar but not equivalent. The first problem (10) can be written as a "weighted" linear problem

$$
\tilde{S}=\sum_{i=1}^{m} w_{i}\left|f_{i} d\left(z_{i}\right)-e_{i} n\left(z_{i}\right)\right|^{2}, \quad w_{i}=\left|e_{i} d\left(z_{i}\right)\right|^{-2} .
$$

The iterative method of Wittmeyer solves the problem iteratively by solving a sequence of linearized problems of the form

$$
\tilde{S}^{(k)}=\sum_{i=1}^{m} w_{i}^{(k)}\left|f_{i} d^{(k)}\left(z_{i}\right)-e_{i} n^{(k)}\left(z_{i}\right)\right|^{2}, \quad k=1,2, \ldots
$$

where $w_{i}^{(k)}=\left|e_{i} d^{(k-1)}\left(z_{i}\right)\right|^{-2}$ uses the denominator $d^{(k-1)}$ from the previous iteration step. Thus using the linearized solution of (11) as a starting value corresponds, e.g., to a choice $d^{(0)}\left(z_{i}\right)=1 / e_{i}, i=1,2, \ldots, m$. Note that this iterative scheme typically does not converge to a minimum of $\tilde{S}$, unless there is no error in the data $[5$, p. 879]. If we set

$$
F_{i}:=\left[f_{i},-e_{i}\right] \quad \text { and } P(z)^{T}:=[d(z), n(z)],
$$

we can rewrite $(11)$ as

$$
S=\sum_{i=1}^{m}\left|F_{i} P\left(z_{i}\right)\right|^{2}=\|P\|^{2}
$$

with the norm defined in (2). We can use the same setup if at each knot $z_{i}$, a vector of complex data is given. So, if $f_{i} \in \mathbb{C}^{1 \times(n-1)}, e_{i} \in \mathbb{C}$, this vector is approximated by a vector rational function, so that also $n(z)$ is an $(n-1)$-dimensional vector polynomial and $d(z)$ a common (scalar) denominator. In the last form of $S$, we then have $F_{i} \in \mathbb{C}^{1 \times n}$ and $P(z) \in \mathbb{C}[z]^{n \times 1}$. Of course we have the degree conditions on $P(z)$, say

$$
\partial P \leq \Delta:=\left[\delta_{1}, \delta_{2}, \ldots, \delta_{n}\right](\text { componentwise }), \quad \Delta \in(\mathbb{N} \cup\{-1\})^{n \times 1}
$$

(we say that the zero polynomial has degree -1 ). To avoid the trivial solution $P \equiv 0$, we add the condition that one of the elements of $P$ has to be monic. Thus, we have precise degree for the monic component. Hence, we consider the following approximation problem.

DEFINITION 6.1 (discrete least squares approximation problem). Given the points $z_{i} \in \mathbb{C}$ and the weight vectors $F_{i} \in \mathbb{C}^{1 \times n}, i=1,2, \ldots, m$, the degree vector $\Delta:=\left[\delta_{1}, \delta_{2}, \ldots, \delta_{m}\right]^{T} \in(\mathbb{N} \cup\{-1\})^{n \times 1}$ and some degree index $\nu_{\Delta} \in\{1,2, \ldots, n\}$. 
With $\bar{\Delta}:=\left(\Delta, \nu_{\Delta}\right)$ (the extended degree vector) and $P:=\left[P_{1}, P_{2}, \ldots, P_{n}\right]^{T} \in$ $\mathbb{C}[z]^{n \times 1}$, consider the sets $\mathcal{P}_{\Delta}$ and $\mathcal{P}_{\bar{\Delta}}$

$$
\begin{aligned}
& \mathcal{P}_{\Delta}:=\left\{P \in \mathbb{C}[z]^{n \times 1} \mid \partial P \leq \Delta\right\}, \\
& \mathcal{P}_{\bar{\Delta}}:=\left\{P \in \mathcal{P}_{\Delta} \mid \partial P_{\nu_{\Delta}}=\delta_{\nu_{\Delta}} \text { and } P_{\nu_{\Delta}} \text { is monic }\right\} .
\end{aligned}
$$

In the discrete least squares approximation problem, we look for the polynomial vector $P$ such that $\|P\|=\min _{Q \in \mathcal{P}_{\bar{\Delta}}}\|Q\|$. It is further assumed that the degree vector $\Delta$ is such that $\mathcal{P}_{\Delta} \subset \mathcal{P}$. Hence, for the set $\mathcal{P}_{\Delta},\|\cdot\|$ is a norm (not a semi-norm).

To solve the discrete least squares approximation problem, we could easily transform it into a linear algebra problem. Note that $F_{i} P\left(z_{i}\right) \in \mathbb{C}$ is a scalar. Therefore, the original problem is equivalent to solving the $m$ linear equations

$$
F_{i} P\left(z_{i}\right)=0, \quad i=1,2, \ldots, m
$$

in a least squares sense, i.e.

$$
\sum_{i=1}^{m}\left|r_{i}\right|^{2} \text { is minimal with } r_{i}=F_{i} P\left(z_{i}\right)
$$

(with $\left.P \in \mathcal{P}_{\bar{\Delta}}\right)$. Because $\mathcal{P}_{\Delta}$ is a $\mathbb{C}$-vector space having dimension $|\Delta|:=\sum_{i=1}^{n}\left(\delta_{i}+\right.$ 1 ), we can choose a basis for $\mathcal{P}_{\Delta}$ and write out the least squares problem using coordinates with respect to this basis. Introducing the normality condition, i.e. $P_{\nu_{\Delta}}$ has to be monic, we can eliminate one of the coordinates. We obtain an $m \times(|\Delta|-1)$ least squares problem. The amount of computational work is proportional to $m|\Delta|^{2}$ (e.g. using the normal equations or the QR factorization).

Assume however that we have an orthonormal basis for $\mathcal{P}_{\Delta}$ such that the basis vectors $B_{j}:=\left[B_{j, 1}, B_{j, 2}, \ldots, B_{j, n}\right]^{T}$ satisfy $\partial B_{j, \nu_{\Delta}}<\delta_{\nu_{\Delta}}, j=1,2, \ldots,|\Delta|-1$, and $\partial B B_{|,|, \nu_{\Delta}}=\delta_{\nu_{\Delta}}$, then we can write every $P \in \mathcal{P}_{\Delta}$ in a unique way as:

$$
P=\sum_{j=1}^{|\Delta|} B_{j} a_{j}, \quad a_{j} \in \mathbb{C} .
$$

Because $P_{\nu_{\Delta}}$ has to be monic of degree $\delta_{\nu_{\Delta}}, a_{|\Delta|}$ is fixed. The other coordinates $a_{j}$, $j=1,2, \ldots,|\Delta|-1$ can be chosen freely. We get

$$
\begin{aligned}
\|P\|^{2} & =\langle P, P\rangle \\
& =\left\langle\sum_{j=1}^{|\Delta|} B_{j} a_{j}, \sum_{j=1}^{|\Delta|} B_{j} a_{j}\right\rangle \\
& \left.=\sum_{j=1}^{|\Delta|}\left|a_{j}\right|^{2} \quad \text { (because }\left\langle B_{i}, B_{j}\right\rangle=\delta_{i j}\right) .
\end{aligned}
$$

Therefore, to minimize $\|P\|$, we can put $a_{j}, j=1,2, \ldots,|\Delta|-1$ equal to zero or

$$
P=B_{|\Delta|} a_{|\Delta|} \text { and } \quad\|P\|=\left|a_{|\Delta|}\right| \text {. }
$$

Hence, to solve the least squares approximation problem we can compute the orthonormal polynomial vector $B_{|\Delta|}$ and this will give us the solution (up to a scalar multiplication to make it monic). In the regular case, the least squares approximation error $\|P\|=\left|a_{|\Delta|}\right|$ is different from zero. 
Suppose we want to solve the problem for a certain degree vector $\Delta^{\star}$. We want to construct the basis vectors for $\mathcal{P}_{\Delta^{\star}}$ recursively by gradually constructing the basis vectors for nested subspaces

$$
\mathcal{P}_{\Delta^{(0)}} \subset \mathcal{P}_{\Delta^{(1)}} \subset \cdots \subset \mathcal{P}_{\Delta^{(k)}}=\mathcal{P}_{\Delta^{\star}} .
$$

If we arrange the degree vectors $\Delta^{(k)}$ into an $n$-dimensional table, then we want to reach $\Delta^{\star}$ by walking along a "diagonal". This means that we pass through the points $\Delta^{\star}-U, \Delta^{\star}-2 U, \ldots$, where $U:=[1,1, \ldots, 1]^{T}$. Each move on the diagonal from $\Delta$ to $\Delta+U$ will be decomposed in a set of $n$ elementary steps in each of the coordinate directions: $\Delta+U_{1}^{1}, \Delta+U_{2}^{1}, \ldots, \Delta+U_{n}^{1}=\Delta+U$, where $U_{j}^{1}:=[1,1, \ldots, 1,0, \ldots, 0]^{T}$ ( $j$ ones). This results in a staircase-like polyline. This works quite well as soon as $\Delta \geq 0$. Unless $\Delta^{\star}$ is on the main diagonal, the starting point of the diagonal through $\Delta^{\star}$ will be outside the positive part of the coordinate system. As soon as some $\delta_{i}<0$, the corresponding polynomial will be zero and it will remain zero, no matter how negative $\delta_{i}$ will get. This means that whenever $\Delta^{(k)}$ falls outside $(\mathbb{N} \cup\{-1\})^{n \times 1}, \mathcal{P}_{\Delta^{(k)}}$ will be equal to some $\mathcal{P}_{\Delta^{(l)}}$ with $\Delta^{(l)} \in(\mathbb{N} \cup\{-1\})^{n \times 1}$. Therefore, we shall project the polyline onto the part $(\mathbb{N} \cup\{-1\})^{n \times 1}$ of $\mathbb{Z}^{n \times 1}$, such that $\Delta^{(k)}<\Delta^{(k+1)}$ for all $k \geq 0$, which means that $\operatorname{dim} \mathcal{P}_{\Delta^{(k+1)}}=\operatorname{dim} \mathcal{P}_{\Delta^{(k)}}+1$, starting with $\Delta^{(0)}=[-1,-1, \ldots,-1]^{T}$, which corresponds to $\mathcal{P}_{\Delta^{(0)}}=\left\{[0,0, \ldots, 0]^{T}\right\}$ with $\operatorname{dim} \mathcal{P}_{\Delta^{(0)}}=0$. Hence, we get the following definition for the sequence of degree vectors $\Delta^{(k)}$, degree indices $\nu_{k}$ and the pivot indices $\pi_{k}, k=1,2, \ldots$

DEFINITION 6.2 (degree vectors, degree indices and pivot indices). Let $\Delta^{\star}:=$ $\left[\delta_{1}^{\star}, \ldots, \delta_{n}^{\star}\right]$ be the target degree vector and define $U_{j}:=[0, \ldots, 0,1,0, \ldots, 0]^{T}(1$ at the $j$-th position). We set the initial degree vector $\Delta^{(0)}:=[-1,-1, \ldots,-1]^{T}$. Furthermore, if $\Delta^{(k-1)}=\left[\delta_{1}, \delta_{2}, \ldots, \delta_{n}\right]^{T}$, then $\Delta^{(k)}:=\Delta^{(k-1)}+U_{j}$ with $j$ the least integer in $\{1,2, \ldots, n\}$ that satisfies the equation $\delta_{j}^{\star}-\delta_{j}=\max \left\{\delta_{i}^{\star}-\delta_{i} \mid i=\right.$ $1,2, \ldots, n\}$. The corresponding degree index is $\nu_{k}:=j$. The pivot indices $\pi_{k}$ are defined as follows. If $\delta_{j}=-1$ then $\pi_{k}:=j$. Otherwise $\pi_{k}:=k-l+n$ with $l$ the number of nonnegative elements in $\Delta^{(k)}$.

By defining the degree vectors in this way, for each degree vector $\Delta^{(k)}>-U$ the degree vector $\Delta^{(k)}-U$ appears earlier in the sequence as $\Delta^{(j)}=\Delta^{(k)}-U$. Also $k-j=n$ is made as small as possible. This will result in a recurrence relation for the orthonormal polynomial vector $\phi_{k}(z)$ written as a linear combination of $z \phi_{j}(z)$ and the other previous orthonormal polynomial vectors. However, when all points $z_{i}$ are on the real line or on the unit circle, only a limited number of the previous orthonormal polynomial vectors will be needed. Hence, the computational work will be decreased by an order of magnitude.

In $[\mathbf{2 1}]$, we have shown that by defining the pivot indices as done in Definition 6.2, Algorithm 2.2 computes the sequence of orthonormal polynomial vectors having the prescribed degree structure.

If we drop the condition that the bilinear form (1) should be a (true) inner product, it can happen that there is an element $P \in \mathcal{P}_{\Delta^{(k)}}$ such that $\|P\|=0$. We call this the singular case. As shown in [21], this element can be found as an orthogonal polynomial vector using the same recurrence relation as before except for the scaling factor. We look for this interpolating solution in the applications of Sections 8 and 9 . 


\section{Data fitting}

In previous publications $[\mathbf{8}, \mathbf{1 9}, \mathbf{2 0}]$, we have considered special cases of the least squares approximation problem described above. In [19], we gave an algorithm to solve the problem with real points $z_{i}, n=2$ and $\delta_{1}=\delta_{2}$. The algorithm is a generalization of the algorithm of Reichel [14], which constructs the optimal polynomial fitting given function values in real points $z_{i}$ in a least squares sense. Reichel's algorithm itself is based on the Rutishauser-Gragg-Harrod algorithm [17, 12, 2] for the computation of Jacobi matrices. Similar results were obtained in [7, 10]. Based on the inverse unitary $Q R$ algorithm for computing unitary Hessenberg matrices [3], Reichel, Ammar and Gragg [15] solve the approximation problem when the given function values are taken in points on the unit circle. In [20], we generalized this from $n=1$ to $n=2$ with equal degrees $\delta_{1}=\delta_{2}$. When $n=2$, we refer the reader to [8], which summarizes [19] and [20] and handles the case of arbitrary degrees $\delta_{1}$ and $\delta_{2}$.

In $[\mathbf{1 9}, \mathbf{2 0}]$, we have given numerical examples showing that the algorithms can be used to compute rational interpolants or rational approximants in a linearized discrete least squares sense.

In this section, we want to focus the attention on the numerical properties of the downdating algorithms. We take the same data as in section 7 of [9]. We consider the following data fitting problem.

$$
\begin{array}{r}
z_{i}=-1+2(i-1) /(M-1)+\delta \\
w_{i}=1 / \sqrt{M}+\delta \\
F_{i}=w_{i}\left[1-\left(1.5+\sin \left(4 x_{i}\right)+\delta\right)\right]
\end{array}
$$

$\delta$ is a uniformly distributed random variable with $|\delta|<10^{-4}$.

We compute the recurrence data to determine a least squares polynomial fit to the noisy data sets $Y_{k}=\left\{z_{i}, F_{i}\right\}_{i=k}^{k+m-1}, k=1,2, \ldots, M-m+1$. So, $m$ is the width of the window sliding over the data. For each $k$, the full recurrence data matrix $D_{k}$ is $m \times(m+n)$. If we consider only the principal $l \times(l+n)$ part during the computations, we denote this by $D_{k}^{(l)}$. To go from step $k$ to step $k+1$, we could first do a downdate by deleting point $k$ and then an update by introducing point $k+m$. Vice versa, we could first update with point $k+m$ and then downdate with point $k$. We shall denote the two variants as DU and UD respectively. There are two possibilities to downdate downdatereal and variantdowndatereal. Let us look at some results now. We shall always take $M=50$. Figures 1 and 2 show the error $\epsilon_{m}$ for $m=5,7,9,11,13,15$ when the complete recurrence data matrix is computed using downdatereal and variantdowndatereal respectively. The error $\epsilon_{j}$ is the 2-norm of the difference of the principal $j \times(j+n)$ part of the computed recurrence data matrix and a reference recurrence data matrix. This reference recurrence data matrix is computed by only updating with the data set $Y_{k}$. Theoretically, the plots of Figures 1 and 2 should be the same! This difference will be much smaller if $l$ is taken smaller than $m$. Note that the error for variantdowndatereal has the same magnitude for all $k$-values except when $m=15$. The plot for $\epsilon_{j}, j<m$, has the same appearance, i.e. the error is determined mainly by the principal part of the recurrence data matrix.

For the following plots, we only keep the principal part $D_{k}^{(l)}$ of the recurrence data matrix, more precisely $l=5$. The number of data points in each data set 


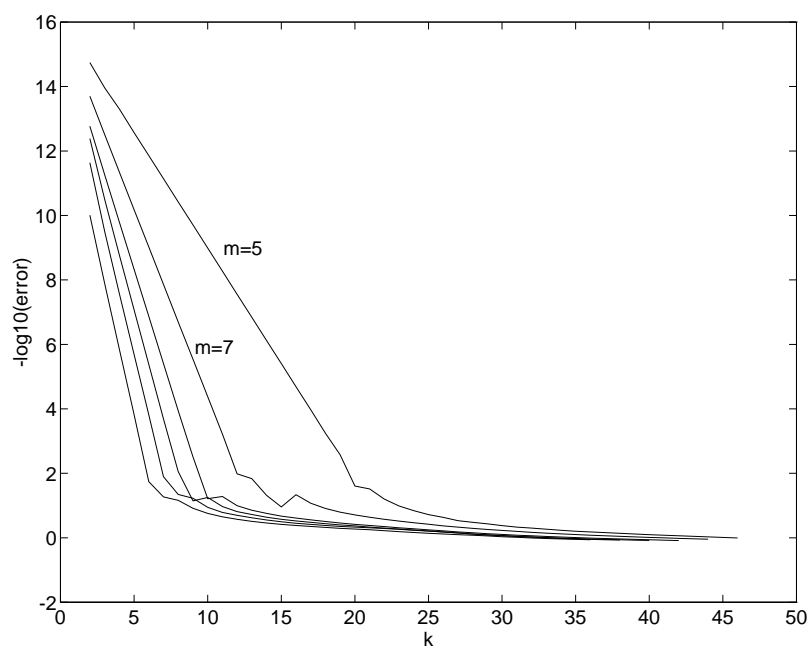

FiguRE 1. $\epsilon_{m}$ using downdatereal for $M=50, m=$ $5,7,9,11,13,15, l=m$

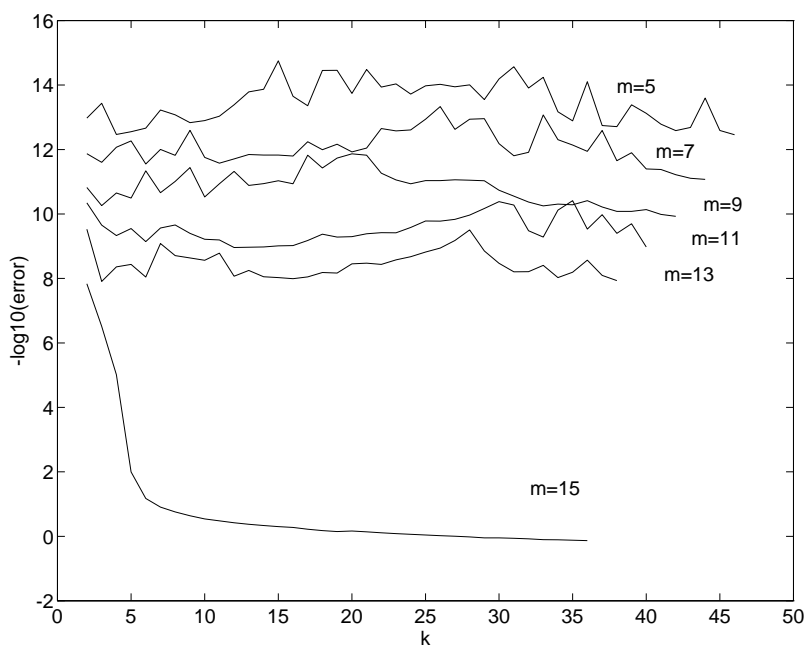

FiguRE 2. $\epsilon_{m}$ using variantdowndatereal for $M=50, m=$ $5,7,9,11,13,15, l=m$

is $m=10$. Figure 3 compares $\epsilon_{2}$ for downdatereal and variantdowndatereal. Characteristicaly, the error $\epsilon_{j}$ for the two cases is only different for small values of $j$. Depending on the (small) random variable $\delta$, there can be peaks or not. Figures 4 and 5 give the errors $\epsilon_{j}, j=1,2,3,4,5$ when downdatereal, respectively variantdowndatereal is used for downdating. In several cases as in Figure 5, we have observed a peak in $\epsilon_{j}$ if variantdowndatereal is used. Occasionally, there is a peak in $\epsilon_{j}$ for downdatereal as in Figure 3. 


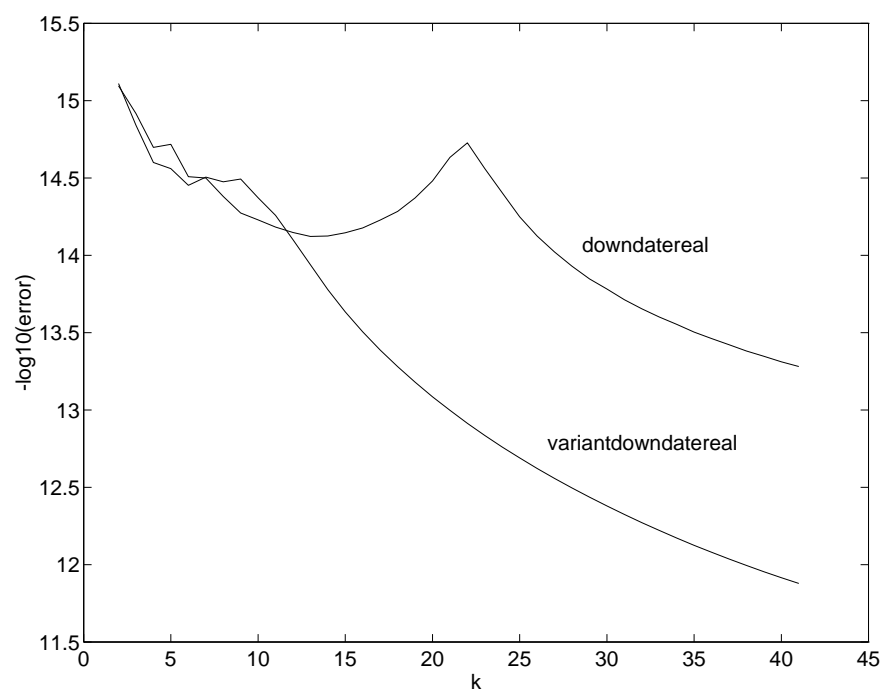

Figure 3. $\epsilon_{2}$ for $M=50, m=10, l=5$

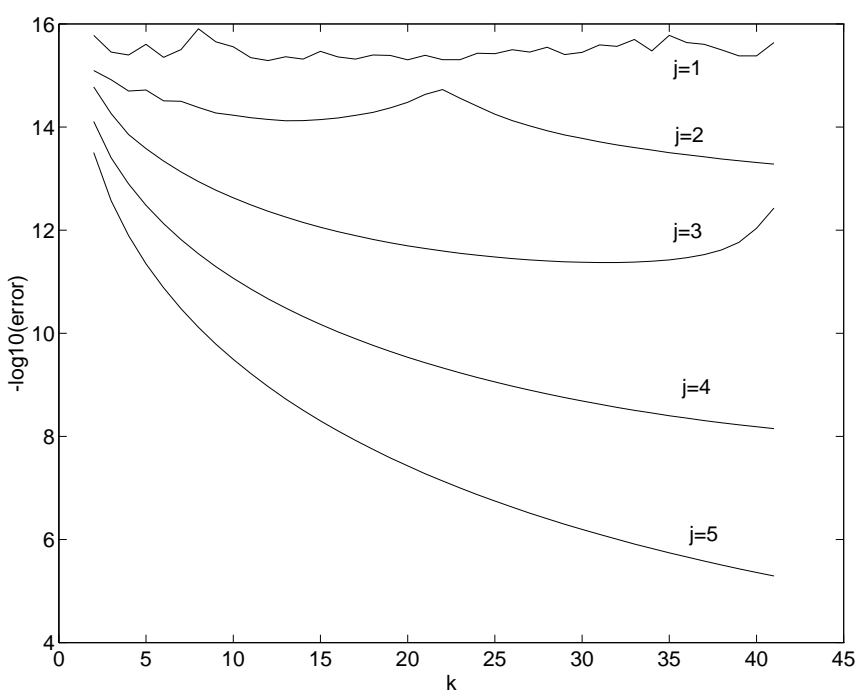

FigURE 4. $\epsilon_{j}, j=1,2,3,4,5$ using downdatereal for $M=50$, $m=10, l=5$

Figure 6 compares the DU and the UD variant of the moving polynomial least squares approximation. We have taken $l=m=10$ and plotted $\epsilon_{m}$. The function variantdowndatereal was used for downdating. If we keep only a part of the recurrence data matrix, the difference between $\epsilon_{j}$ of UD and DU is small for small $j$ and very small if $j$ increases. This is true for downdatereal as well as for variantdowndatereal. 


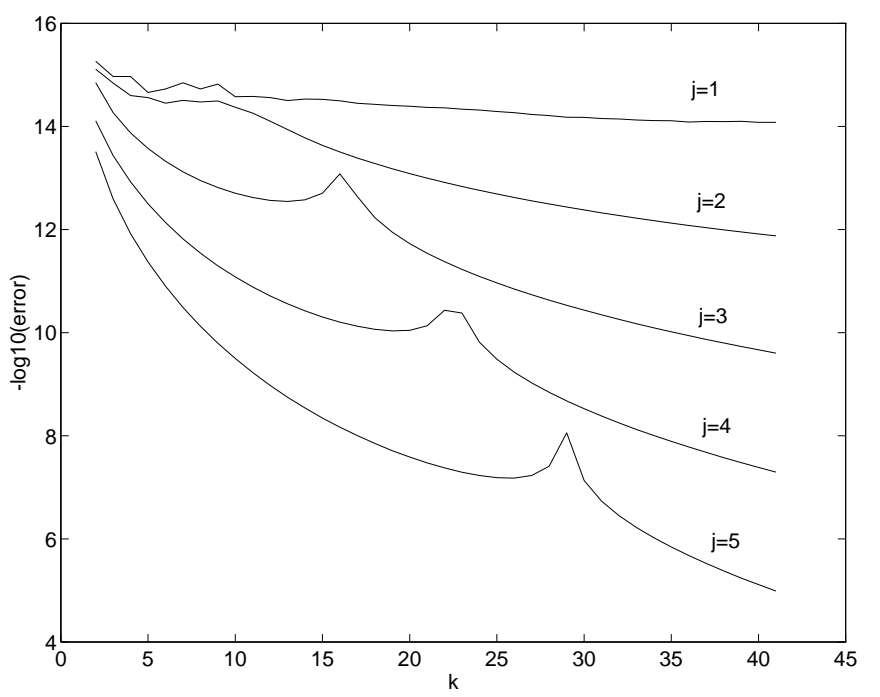

FIGURE 5. $\epsilon_{j}, j=1,2,3,4,5$ using variantdowndatereal for $M=50, m=10, l=5$

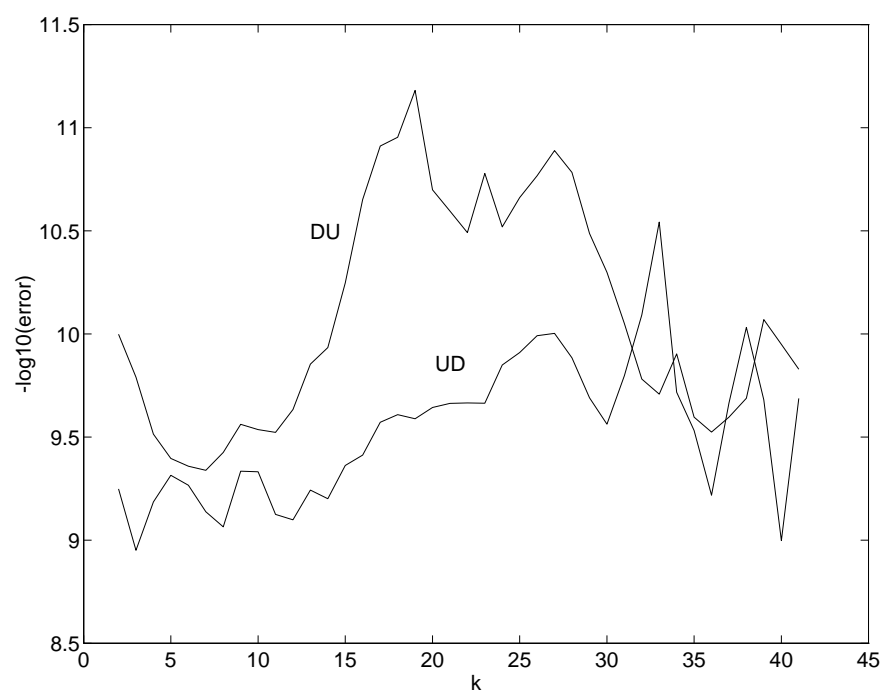

FigURE 6. $\epsilon_{m}$ for $M=50, m=l=10$

When computing the partial recurrence data matrix for $l=7,8,9$, the norm of $p$ became bigger than 1 when using downdatereal. Hence, $\eta$ could not be computed. This did not happen for variantdowndatereal.

In Figures 7 and $8, \epsilon_{m}$ is plotted keeping $k=1$ and $m=10$ fixed where we update $D_{k}$ with the $i$ data points $z_{k+j}, F_{k+j}, j=1,2, \ldots, i$ and then downdate again using the same data points. Figure 7 shows the results when we downdate first with the data point $z_{k+i}, F_{k+i}$, then with $z_{k+i-1}, F_{k+i-1}$ and so on. Figure 8 plots $\epsilon_{m}$ when we downdate the data points in reversed order. The ordering of 


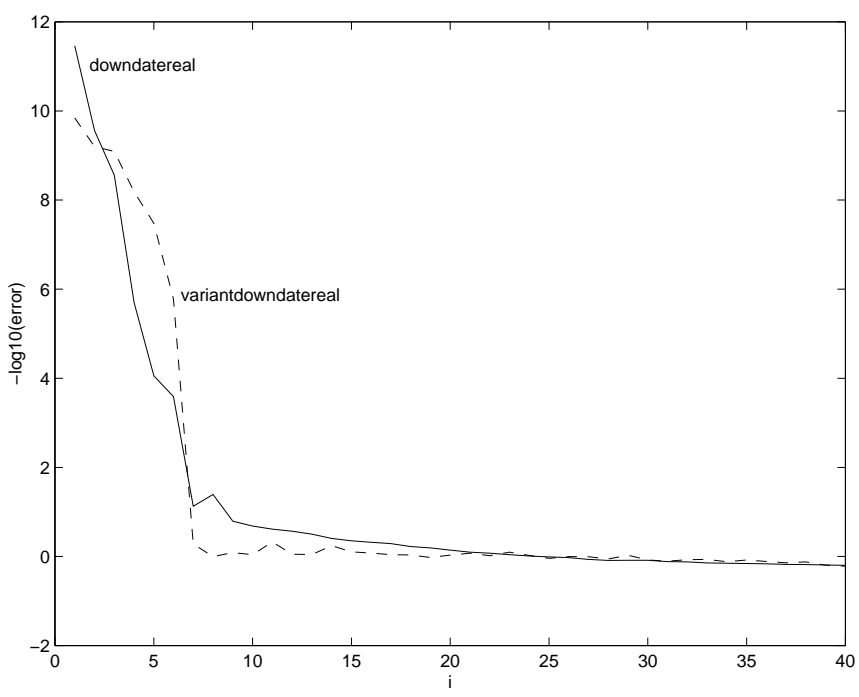

FiguRe 7. $\epsilon_{m}$ for $M=50, m=l=10$

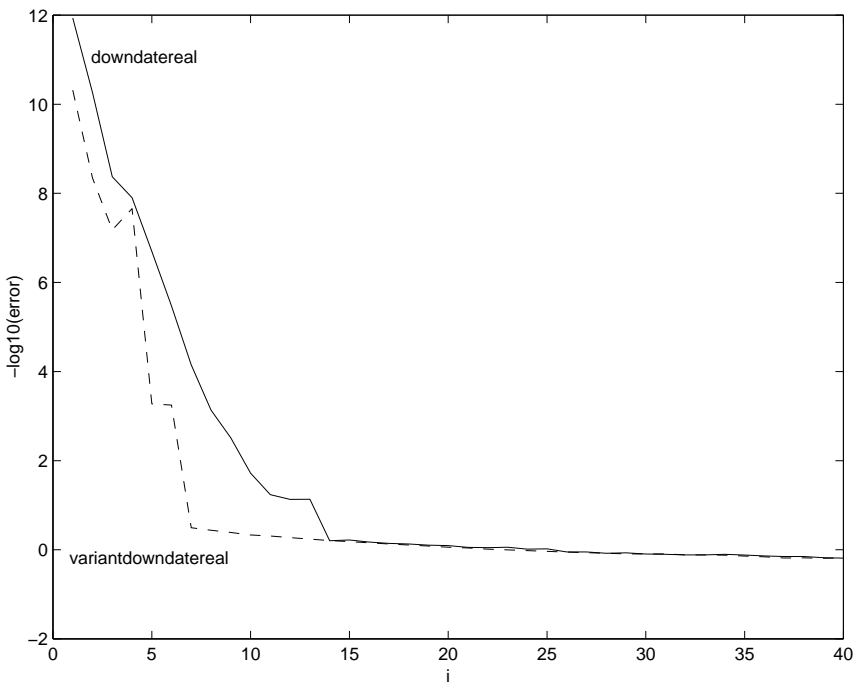

FiguRe 8. $\epsilon_{m}$ for $M=50, m=l=10$

the data points when updating does not influence the accuracy much but the two Figures 7 and 8 indicate the importance of the ordering when downdating. They also show that updating with a number of data points and then downdating with the same points leads to a significant loss of precision in the recurrence data matrix.

The results of this section are very similar to those obtained by Elhay, Golub and Kautsky in [9]. 


\begin{tabular}{|r|r|}
\hline $7.216118463251689 \mathrm{e}-01$ & $+9.643808820429823 \mathrm{e}-01 i$ \\
$7.216118463251689 \mathrm{e}-01$ & $-9.643808820429823 \mathrm{e}-01 i$ \\
$-1.313967055995811 \mathrm{e}+00$ & $+1.377388260335852 \mathrm{e}-01 i$ \\
$-1.313967055995811 \mathrm{e}+00$ & $-1.377388260335852 \mathrm{e}-01 i$ \\
$1.184710419341417 \mathrm{e}+00$ & \\
\hline
\end{tabular}

TABLE 1. The computed zeros of the polynomial system

\section{Solving a system of two polynomial equations in two variables}

The results of this section are based on an idea of P. Verlinden and A. Haegemans $[\mathbf{2 2}]$. The system of two equations which we solve here is

$$
\begin{gathered}
f(x, z)=z x^{2}+2 x-1=0 \\
g(x, z)=x-z^{2}+1=0 .
\end{gathered}
$$

Let us consider the function

$$
R(z)=\prod_{\bar{x}} g(\bar{x}, z)
$$

where the product is taken over all possible solutions $\bar{x}$ of $f(x, z)=0$. This function $R(z)$ is a rational function. Its zeros give the $z$-component of all solutions of the original system of polynomial equations. The 40 points $z_{i}$ were taken on the real line equidistant in the interval $[-2,2]$. The computed zeros of $R(z)$ based on the 11-th "orthonormal" polynomial vector are given in Table 1. The first two zeros are accurate except for the last four digits shown while the other zeros are accurate except for the two last decimal digits shown. Because the data points $z_{i}$ are further away from the first two zeros than from the other zeros, the accuracy for the first two zeros is a little bit lower. This can be remedied by choosing the data points in the neighbourhood of these zeros.

\section{Solution of the Bezout equation}

We compute the recurrence coefficients for two polynomials $x(z)$ and $y(z)$ satisfying the Bezout equation

$$
a(z) x(z)+b(z) y(z)=1, \quad \forall z \in \mathbb{C}
$$

where $a(z)$ and $b(z)$ are given polynomials. We assume without loss of generality that $\operatorname{deg} a \leq \operatorname{deg} b$. The difference $\operatorname{deg} x-\operatorname{deg} y=\operatorname{deg} b-\operatorname{deg} a=: \delta$ determines the degree structure of the orthonormal polynomial vectors $\phi(z)$, i.e. the pivot indices $\pi_{j}=1,4,5, \ldots, \delta+3,2, \delta+4, \ldots$.

In Figure 9 we show the residual $r(z):=a(z) x(z)+b(z) y(z)-1$ on the unit circle and on the real line for a polynomial $a(z)$ of degree 10 and $b(z)$ of degree 15 (both with random coefficients). On the real line, it is a kind of extrapolation because the data points were taken on the real line in the interval $[-2,2]$ for 50 equidistant points.

\section{Conclusion}

In this paper, we give updating and downdating procedures to compute the recurrence information for sequences of polynomial vectors orthonormal with respect to a discrete inner product. We have shown the usefulness of these orthonormal 

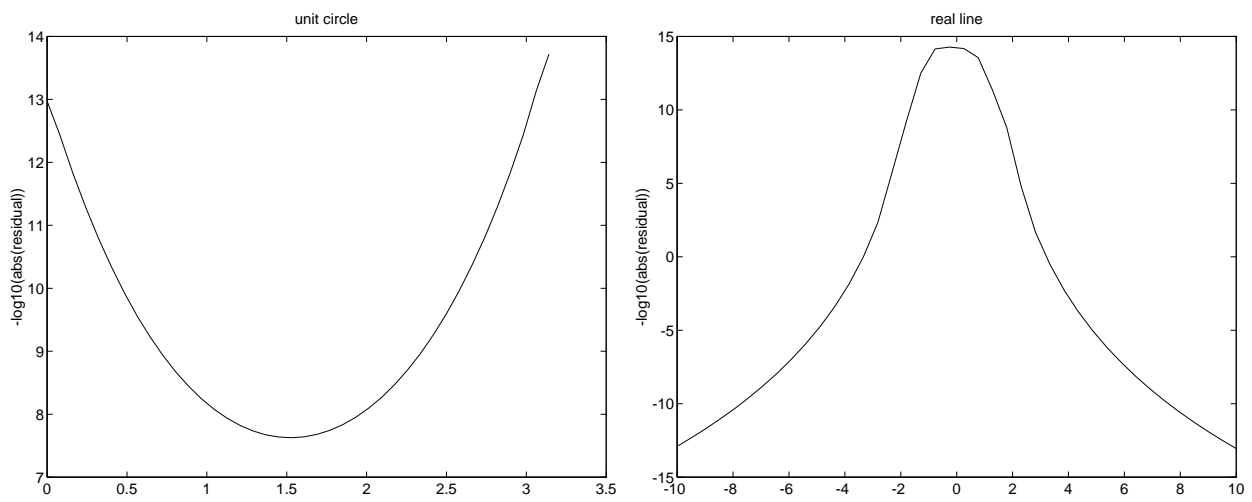

FiguRE 9. residual $r(z)$

polynomial vectors in a number of applications. When only updating is used, we obtain accurate results but if also downdating is involved there is a decrease in accuracy. How to limit this decrease is still an open question. The details of the downdating procedure are important as we have shown by comparing downdate and variantdowndate. Also the ordering in which the donwdating is done plays an important factor as was shown in Figures 7 and 8.

\section{References}

[1] G. Ammar and C. He, On an inverse eigenvalue problem for unitary Hessenberg matrices, Linear Algebra Appl. 218 (1995), 263-272.

[2] G.S. Ammar and W.B. Gragg, $O\left(n^{2}\right)$ reduction algorithms for the construction of a band matrix from spectral data, SIAM J. Matrix Anal. Appl. 12 (1991), 426-431.

[3] G.S. Ammar, W.B. Gragg, and L. Reichel, Constructing a unitary Hessenberg matrix from spectral data, Numerical linear algebra, digital signal processing and parallel algorithms (Berlin) (G. Golub and P. Van Dooren, eds.), NATO-ASI Series, F: Computer and Systems Sciences, vol. 70, Springer, 1991, pp. 385-395.

[4] _ Downdating of Szegő polynomials and data-fitting applications, Linear Algebra Appl. 172 (1992), 315-336.

[5] I. Barrodale and J.C. Mason, Two simple algorithms for discrete rational approximation, Math. Comp. 24 (1970), 877-891.

[6] D.L. Boley and G.H. Golub, A survey of matrix inverse eigenvalue problems, Inverse problems (Bristol, England), vol. 3, Physics Trust Publications, 1987, pp. 595-622.

[7] _ A survey of matrix inverse eigenvalue problems, Inverse Problems 3 (1987), 595-622.

[8] A. Bultheel and M. Van Barel, Vector orthogonal polynomials and least squares approximation, SIAM J. Matrix Anal. Appl. 16 (1995), no. 3, 863-885.

[9] S. Elhay, G.H. Golub, and J. Kautsky, Updating and downdating of orthogonal polynomials with data fitting applications, SIAM J. Matrix Anal. Appl. 12 (1991), 327-353.

$[10]$, Updating and downdating of orthogonal polynomials with data fitting applications, SIAM J. Matrix Anal. Appl. 12 (1991), 327-353.

[11] W. B. Gragg and W. J. Harrod, The numerically stable reconstruction of Jacobi matrices from spectral data, Numer. Math. 44 (1984), 317-335.

[12], The numerically stable reconstruction of Jacobi matrices from spectral data, Numer. Math. 44 (1984), 317-335.

[13] T. Pomentale, On rational discrete least squares approximation, Numer. Math. 12 (1968), $40-46$.

[14] L. Reichel, Fast $Q R$ decomposition of Vandermonde-like matrices and polynomial least squares approximation, SIAM J. Matrix Anal. Appl. 12 (1991), 552-564. 
[15] L. Reichel, G.S. Ammar, and W.B. Gragg, Discrete least squares approximation by trigonometric polynomials, Math. Comp. 57 (1991), 273-289.

[16] H. Rutishauser, On Jacobi rotation patterns, Proceedings of Symposia in Applied Mathematics, vol. 15 , Experimental Arithmetic, High Speed Computing and Mathematics (Providence), Amer. Math. Soc., 1963, pp. 219-239.

[17] _ On Jacobi rotation patterns, Proceedings of Symposia in Applied Mathematics, vol. 15, Experimental Arithmetic, High Speed Computing and Mathematics (Providence), Amer. Math. Society, 1963, pp. 219-239.

[18] P. Spellucci, Algorithms for rational discrete least squares approximation , Part I: unconstrained optimization, Mod. Meth. Numer. Math., Tag. Clausthal 1975, ISNM 32, 1976, pp. 139-157.

[19] M. Van Barel and A. Bultheel, A parallel algorithm for discrete least squares rational approximation, Numer. Math. 63 (1992), 99-121.

[20] Discrete linearized least squares approximation on the unit circle, J. Comput. Appl. Math. 50 (1994), 545-563, Special Issue Proceedings of the International Conference on Computational and Applied Mathematics, Leuven, Aug., 1992.

[21] _ Orthonormal polynomial vectors and least squares approximation for a discrete inner product, Electron. Trans. Numer. Anal. 3 (1995), 1-23.

[22] P. Verlinden and A. Haegemans, Solving very deficient systems of polynomial equations by implicit elimination, Tech. Report TW207, Dept. of Computer Science, K.U.Leuven, May 1994.

[23] L. Wittmeyer, Rational approximation of empirical functions, Nordisk Tidskr. Informationsbehandling 2 (1962), 53-60.

Department of Computer Science, K.U.Leuven, Celestijnenlaan 200 A, 3001 HeverLEE, BELGIUM

E-mail address: Marc.VanBarel@cs.kuleuven.ac.be

$U R L:$ http://www.kuleuven.ac.be/ $\sim \operatorname{marc} /$

Department of Computer Science, K.U.Leuven, Celestijnenlaan 200 A, 3001 HeverLEE, BELGIUM

E-mail address: Adhemar.Bultheel@cs.kuleuven.ac.be

$U R L:$ http://www.kuleuven.ac.be/ ade/ 